Short Communication

\title{
Real-Time SARS-CoV-2 Genotyping by High-Throughput Multiplex PCR Reveals the Epidemiology of the Variants of Concern in Qatar
}

\author{
Mohammad R. Hasan ${ }^{1,5, *}$, Mahesh K.R. Kalikiri², Faheem Mirza1, Sathyavathi Sundararaju1, \\ Anju Sharma ${ }^{1}$, Thabisile Xaba ${ }^{1}$, Stephan Lorenz ${ }^{2}$, Hiam Chemaitelly ${ }^{3,4}$, \\ Reham A. El-Kahlout ${ }^{6}$, Kin Ming Tsui ${ }^{1,5}$, Hadi M. Yassine ${ }^{7}$, Peter V. Coyle ${ }^{6,7,9}$, Abdullatif Al \\ Khal $^{6}$, Roberto Bertollini ${ }^{8}$, Mohamed H. Al Thani ${ }^{8}$, Laith J. Abu-Raddad ${ }^{3,4}$, Patrick Tang ${ }^{1,5}$, \\ National Study Group for COVID-19 Epidemiology in Qatar
}

\footnotetext{
${ }^{1}$ Department of Pathology, Sidra Medicine, Doha, Qatar

${ }^{2}$ Department of Research, Sidra Medicine, Doha, Qatar

${ }^{3}$ Infectious Disease Epidemiology Group, Doha, Qatar Doha, Qatar

${ }^{5}$ Pathology and Laboratory Medicine, Weill Cornell Medicine-Qatar, Doha, Qatar

${ }^{6}$ Hamad Medical Corporation, Doha, Qatar

${ }^{7}$ Biomedical Research Center, Qatar University, Doha, Qatar

${ }^{8}$ Ministry of Public Health, Doha, Qatar

${ }^{9}$ Welcome-Wolfson Institute for Experimental Medicine, Queens University, Belfast, UK
}

${ }^{4}$ World Health Organization Collaborating Centre for Disease Epidemiology Analytics on HIV/AIDS, Sexually Transmitted Infections, and Viral Hepatitis,

\section{A R T I C L E I N F O}

\section{Article history:}

Received 5 August 2021

Revised 1 September 2021

Accepted 3 September 2021

\section{Keywords:}

COVID-19

SARS-CoV-2

Variants of concern (VOC)

Genotyping

Epidemiology

\begin{abstract}
A B S T R A C T
Complementing whole genome sequencing strategies with high-throughput multiplex RT-qPCR genotyping allows for more comprehensive and real-time tracking of SARS-CoV-2 variants of concern. During the second and third waves of COVID-19 in Qatar, PCR genotyping, combined with Sanger sequencing of untypeable samples, was employed to describe the epidemiology of the Alpha, Beta and Delta variants. A total of 9792 nasopharyngeal PCR-positive samples collected between April-June 2021 were successfully genotyped, revealing the importation and transmission dynamics of these three variants in Qatar.

(c) 2021 The Author(s). Published by Elsevier Ltd on behalf of International Society for Infectious

Diseases.

This is an open access article under the CC BY-NC-ND license (http://creativecommons.org/licenses/by-nc-nd/4.0/)
\end{abstract}

The emergence of severe acute respiratory syndrome coronavirus-2 (SARS-CoV-2) variants of concern (VOC) has threatened the extraordinary public health and vaccination efforts against coronavirus disease 2019 (COVID-19). These variants, which include Alpha, Beta, Gamma and Delta (Pango lineage B.1.1.7, B.1.351, P.1 and B.1.617.2, respectively), have now spread to many countries around the world (WHO, 2021). Therefore, in addition to the routine surveillance for SARS-CoV-2, it is important to monitor the epidemiology of these VOCs to determine the effectiveness of vaccines (Abu-Raddad et al., 2021a; Chemaitelly et al., 2021) and public health measures to prevent their spread.

\footnotetext{
* Corresponding author: Mohammad Rubayet Hasan, PhD, D(ABMM), FCCM. Clinical Molecular Microbiologist, Pathology Sciences, Department of Pathology, Sidra Medicine, Level 2M, Office H2M-24093, PO BOX 26999, Doha, Qatar. Tel.: +974 4003 2996; +97430035501.

E-mail address: mhasan@sidra.org (M.R. Hasan).
}

This study employed a previously described multiplex RT-qPCR genotyping strategy targeting the $\triangle 69 / 70 \mathrm{HV}$ deletion in the spike gene and the $\triangle 3675-3677$ SGF deletion in the ORF1a gene to distinguish the Alpha variant from Beta/Gamma variants (Vogels et al., 2021). The method also included CDC N1 assay to identify 'other' non-Alpha and non-Beta/Gamma variants for further subtyping. Multiplex RT-qPCR was performed, and the results were interpreted with minor modification of the original method. With the aid of liquid handling robotics, the previously described strategy was adapted to scale-up variant screening capacity. To characterize the 'other' category, sequencing of the N-terminal and receptorbinding (RBD) regions of the SARS-CoV-2 surface glycoprotein (S) gene was performed (Supplementary data).

Between 27 March and 19 June 2021, a total of 13,797 randomly selected SARS-CoV-2-positive NPS specimens were received on a weekly basis from the national COVID-19 biorepository at the 
A)

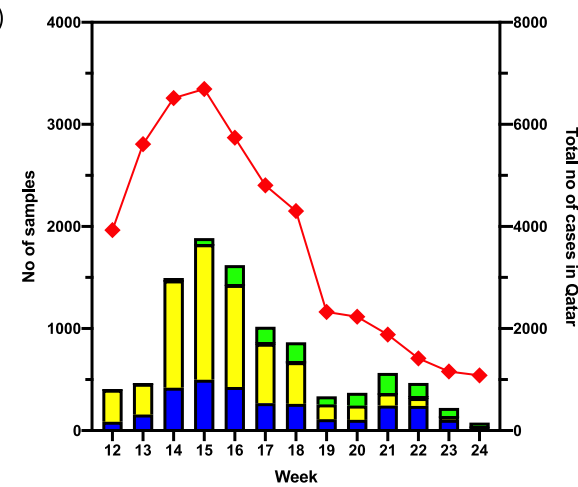

C)

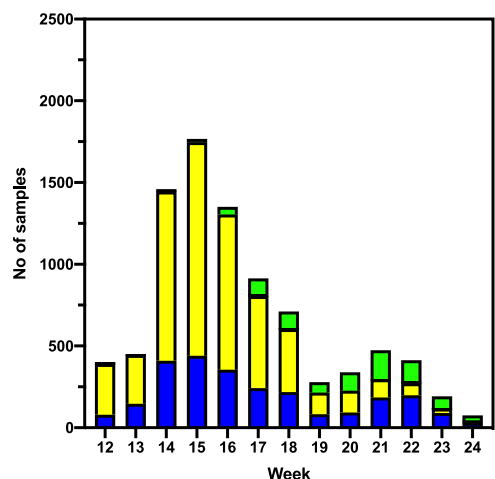

B)

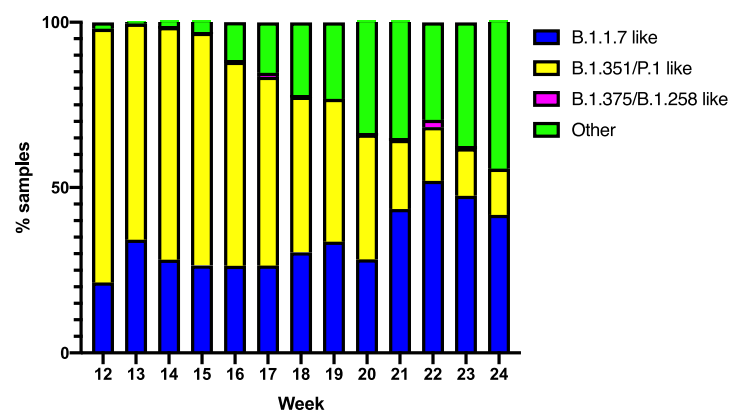

D)

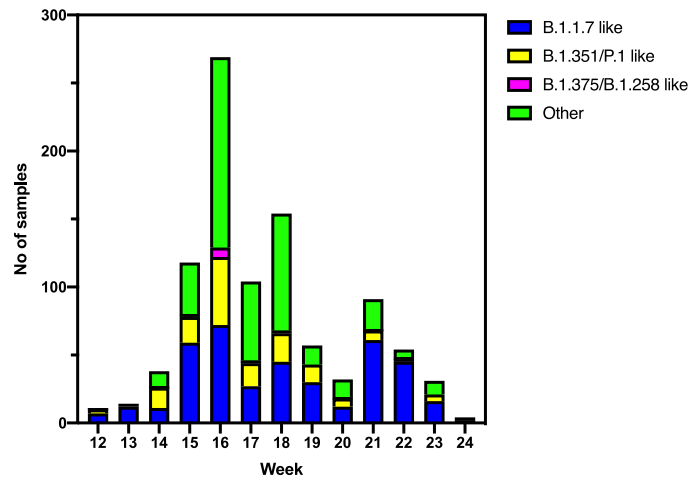

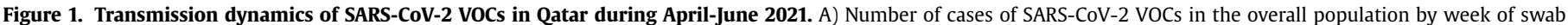

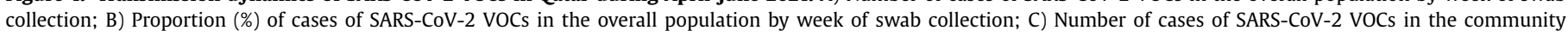
by week of swab collection; D) Imported cases of SARS-CoV-2 VOCs by week of swab collection.

Qatar Biobank. The number of samples that were received ranged from approximately $10 \%-47 \%$ of the total number of new positive cases reported in the country each week (Figure 1A) (https:// www.data.gov.qa/pages/home/). A total of 9792 samples were successfully genotyped by the variant PCR, and 4005 samples remained undetermined because of weak or undetermined RT-qPCR Ct values. Overall, 2970 (30.3\%) were B.1.1.7-like, 5500 (56.2\%) were B.1.351/P.1-like, 40 (0.4\%) were B.1.375/B.1.258-like and 1282 (13.1\%) were 'other' variants. Genotyping data were verified by sequencing a total of 236 random NPS samples (Supplemental methods). PCR genotyping results were $88.8 \%, 99.5 \%$ and $100 \%$ in agreement with the SARS-CoV-2 lineage assignment by sequencing for B.1.1.7like, B.1.351/P.1-like and 'other' variants, respectively. Additionally, a total of 367 random NPS samples from the 'other' category were sequenced (Supplemental methods), of which 354 (96.5\%) were B.1.617.2-like, seven (1.9\%) were B.1.617.1-like, three (0.8\%) were B.1-like, one $(0.3 \%)$ was B.1.617.3-like and one (0.3\%) remained undetermined.

In order to describe the epidemiology of the VOCs, the collection date, age, sex, nationality, reason for testing, and vaccination status associated with each sample were extracted from a federated, national SARS-CoV-2 database. The second and third waves of the COVID-19 pandemic in Qatar were predominantly driven by the spread of the B.1.1.7 and B.1.351 variants, respectively (Chemaitelly et al., 2021). The large third wave, dominated by the B.1.351 variant, led to the large contribution of B.1.351/P.1like cases in the multiplex PCR testing. As the epidemic began to decline in Qatar, large numbers of cases with 'other' variants were detected (Figures 1A and 1B). The epidemiology of the SARS-CoV2 VOCs in the community was similar to the overall epidemiology (Figure 1C). However, a large number of B.1.1.7-like and 'other' variants were detected from the imported cases coincident with the outbreaks in India and Nepal (Figure 1D) and the importation of the 'other' variant significantly declined after targeted travel restrictions were implemented during week 17.

The 'other' variant, which was mostly the 'Delta' variant, was initially seen within those of Indian nationality, but it eventually spread to other nationalities (Figure 2A). Notably, despite the influx of the Delta variant into the community, it was unable to generate a significant outbreak. This is likely due to the high level of immunity in the community from the mass vaccination campaign and previous infection (Abu-Raddad et al., 2021b), as well as the continuation of various public health measures against the spread of SARS-CoV-2. For each variant, most cases during the second and third waves were in individuals aged between 25-44 years and in those who were not fully vaccinated (Figure 2B, Supplemental Figure 1). With the decline of the epidemic in Qatar to a low-incidence phase, the resulting distribution of the three dominant variants (Alpha, Beta, and Delta) suggests a higher effective reproduction number or transmissibility of Alpha and Delta variants, which makes them more successful versus the Beta variant (Campbell et al., 2021).

In conclusion, this study demonstrated that actionable epidemiological data can be generated by variant PCR so that important public health decisions can be made in a timely manner. In particular, for countries lacking the technical capability and resources for large-scale surveillance based on sequencing, variant PCR may be a useful tool in informing national COVID-19 responses and preventing the importation of SARS-CoV-2 VOCs and reducing their spread in the community.

\section{Author contributions}

MRH conceived the idea, co-designed the study, analyzed data and wrote the first draft of the manuscript. MKRK, FM, SS, and AS performed experiments and analyzed data. PT co-designed the 
A)

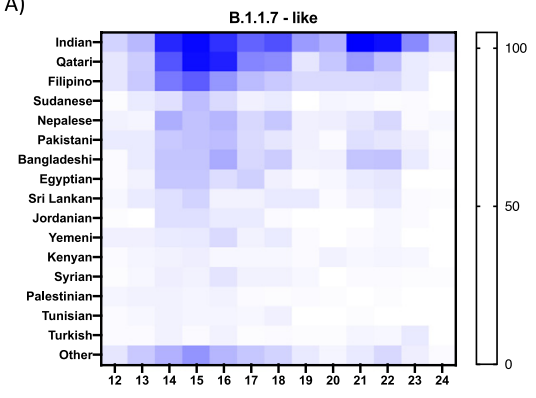

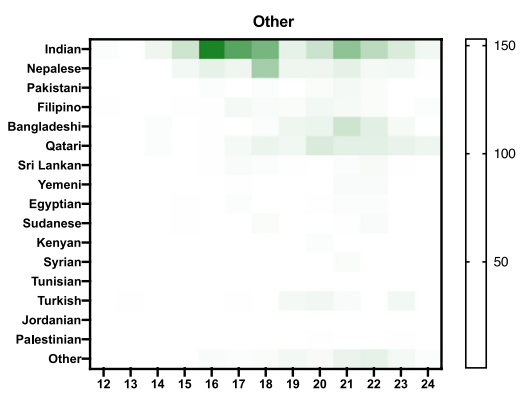

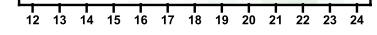

B)

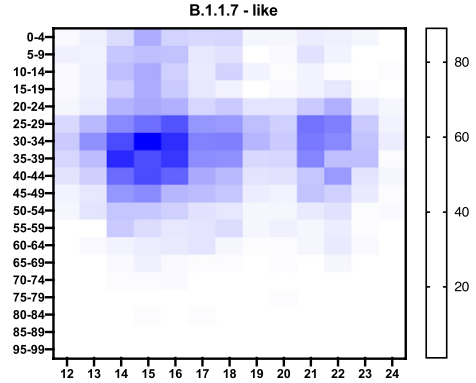

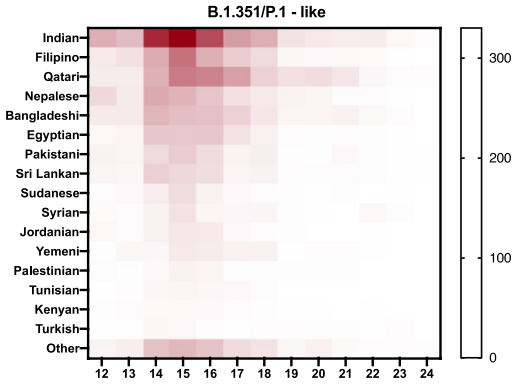

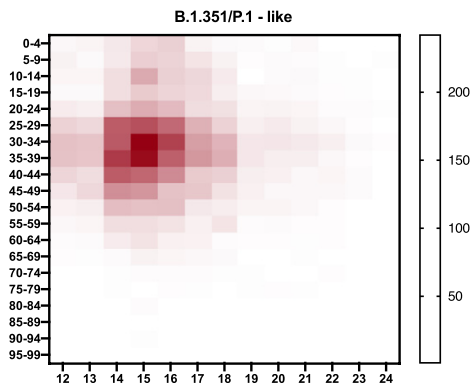

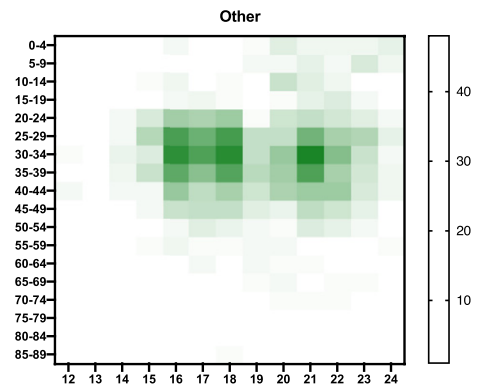

Figure 2. Distribution of cases with SARS-CoV-2 VoCs. A) Number of cases of SARS-CoV-2 VOCs by nationality; B) Number of cases of SARS-CoV-2 VOCs by age.

study and contributed to the writing of the manuscript. All other authors contributed to data collection and acquisition, database development, and critical review of the manuscript. All authors have read and approved the final manuscript.

\section{Conflict of Interest/Disclosure}

The authors have declared no conflicts of interest.

\section{Funding}

This research did not receive any specific grant from funding agencies in the public, commercial, or not-for-profit sectors.

\section{Ethical Approval}

The study was approved by the Hamad Medical Corporation Institutional Review Board with waiver of informed consent.

\section{References}

Abu-Raddad LJ, Chemaitelly H, Butt AA. National Study Group for Covid-19 Vaccination. Effectiveness of the BNT162b2 Covid-19 Vaccine against the B.1.1.7 and B.1.351 Variants. N Engl J Med 2021a.

Abu-Raddad LJ, Chemaitelly H, Coyle P, Malek JA, Ahmed AA, Mohamoud YA, et al. SARS-CoV-2 antibody-positivity protects against reinfection for at least seven months with 95\% efficacy. E Clin Med 2021b;35.

Campbell F, Archer B, Laurenson-Schafer H, Jinnai Y, Konings F, Batra N, et al. Increased transmissibility and global spread of SARS-CoV-2 variants of concern as at June 2021. Euro Surveill 2021;26(24).

Chemaitelly H, Yassine HM, Benslimane FM, Al Khatib HA, Tang P, Hasan MR, et al. mRNA-1273 COVID-19 vaccine effectiveness against the B.1.1.7 and B.1.351 variants and severe COVID-19 disease in Qatar. Nat Med 2021.

Vogels C, Fauver J, Grubaugh N. Multiplexed RT-qPCR to screen for SARS-COV-2 B.1.1.7, B.1.351, and P.1 variants of concern V.3. protocolsio 2021.

WHO. Tracking SARS-CoV-2 variants; 2021. Available from: https://www.who.int/en/ activities/tracking-SARS-CoV-2-variants/. (Accessed 24 June 2021). 